\title{
Editorial commentary on Indian Journal of Gastroenterology—July-August 2021
}

\author{
Jimmy K. Limdi ${ }^{1,2,3}$ (B) \\ Published online: 16 June 2021 \\ (C) Indian Society of Gastroenterology 2021

\section{Quality of life 5 years following liver transplantation}

Liver transplantation (LT) in well-selected patients has had a transformational effect on people with end-stage liver disease and acute liver failure [1]. Quality of life (QoL) after LT, however, is a function of several determinants, including level of education, understanding of disease, complexity of medical prescriptions and family support; but data are scarce. Raju and colleagues from the Amrita Institute of Medical Sciences, Kochi, India, report a prospective study on QoL and incidence of complications 5-years following LT [2]. Data on the incidence of new metabolic complications and renal dysfunction were assessed from 130 patients who completed at least 5 years of follow-up after LT. The LT Database questionnaire was administered to 100 patients. New-onset dyslipidemia, diabetes, renal dysfunction, hypertension and overweight/ obesity were noted in 43\%, 26.7\%, 25\%, $16.4 \%$ and $15.4 \%$, respectively. Significant improvement was noted in psychological status, personal function and social and general health perceptions as also measures of disease activity. Prospective data from centres involved in LT, feeding into a National Registry, may be needed to record, assess and improve standards of care and outcomes.

Jimmy K. Limdi

Jimmy.Limdi@nhs.net

1 Section of Inflammatory Bowel Diseases, Division of Gastroenterology, The Pennine Acute Hospitals NHS Trust, Manchester, UK

2 Manchester Academic Health Sciences, University of Manchester, Manchester, UK

3 Manchester Metropolitan University, Manchester, UK

\section{Transjugular intrahepatic portosystemic shunt placement at index portal hypertensive decompensation (anticipant-TIPS) in cirrhosis and the role of early intervention in variceal bleeding and ascites}

Ascites or bleeding from varices (and recurrence) as the first sign of portal hypertensive (PHT) decompensation is associated with poor survival [3]. Beta-blockers are effective in the reduction of hepatic venous pressure gradient (HVPG) from baseline by $12 \%$ at 1 year, decreasing to $10 \%$ by 3 years. In contrast, transjugular intrahepatic portosystemic shunt (TIPSS) placement can reduce portal pressure by $70 \%$ from baseline [4]. In an elegant study from the Ernakulam Medical Centre, Kochi, India, reported in this issue of the Indian Journal of Gastroenterology, the investigators studied clinical, biochemical and liver disease severity parameters and survival, at baseline and after intervention, in patients with cirrhosis, undergoing TIPSS at the first significant PHT decompensation (anticipant-TIPSS), compared to those undergoing TIPSS for recurrent or persistent PHT complications, as compared to a former group of matched historical controls on standard medical therapy [5]. Conventional TIPSS was associated with increased length of stay on intensive care post-procedure, re-admission and sepsis. Patients undergoing anticipant TIPSS had a low rate of hospitalization, recurrence of varices and ascites at 1 year, with reduced hospital visits and better 1-year overall and sub-grouped survival compared to those receiving standard medical therapy. These findings are worthy of further exploration in carefully designed prospective studies.

\section{Rodenticide ingestion is an important cause of acute hepatotoxicity in Tamil Nadu, southern India}

Rodenticide-induced hepatotoxicity is a major cause of acute liver failure (ALF) in southern and western India 
[6]. Yellow phosphorus is a protoplasmic toxin with no specific antidote (unlike $\mathrm{N}$-acetyl cysteine for paracetamol overdose, a major source of ALF in the western world). Management is mainly supportive and may require liver transplantation. The Tamil Nadu Chapter of the Indian Society of Gastroenterology reports a large retrospective series of acute hepatotoxicity caused by hepatotoxins including rodenticides and paracetamol [7]. Of 685 patients consuming rodenticide, hepatotoxicity was observed in 450 patients; 396 were managed conservatively, 54 required plasma exchange and another patient required plasma exchange and subsequently a liver transplant. A sobering observation was that a third $(35 \%)$ of patients had an adverse outcome; 131 died and 28 were discharged in a moribund state.

The study serves as a "wake-up" call for improved preventative care through tighter regulation of access to rodenticide and improved public knowledge and awareness of risks, as indeed improved triage and access to emergency and critical care services. This study should serve as an impetus for urgent data from other regions in India.

\section{Comparison of clinical and metabolic profiles of lean versus non-lean non-alcoholic fatty liver disease}

Non-alcoholic fatty liver disease (NAFLD) is a growing public health problem. The strong association between NAFLD and metabolic complications such as type 2 diabetes, cardiovascular disease, chronic kidney disease and some types of extrahepatic malignancies is well recognized [8]. It is also acknowledged that NAFLD can occur in non-obese individuals. Navarroza and Wong from the University of Santo Tomas and Chinese General Hospitals, Philippines, report a crosssectional retrospective study of 663 patients with NAFLD [9]. Their aim was to compare clinical and biochemical profiles of non-lean vs. lean $(\mathrm{BMI}<23)$ individuals with NAFLD. Non-lean patients were more likely to have hypertension, diabetes mellitus, dyslipidemia and the metabolic syndrome with transaminase elevations. Metabolic derangements were noted in a third of lean patients who comprised $11.9 \%$ of the NAFLD group. Older age, low albumin, a high BARD (BMI, AST/ALT Ratio, T2 diabetes mellitus) score and symptoms were all independently associated with the risk of cirrhosis or hepatocellular carcinoma. The authors underscore the importance of early recognition and characterization of NAFLD even in lean individuals and the need for further research in this area.
Hepatocellular carcinoma patients with portal vein thrombosis treated with robotic radiosurgery: Interim results of a prospective study

Although treatment outcomes for hepatocellular carcinoma (HCC) with focal resection, radiofrequency ablation, transarterial chemoembolization or stereotactic body radiotherapy (SBRT) are encouraging when associated with preserved liver function, associated portal vein thrombosis (PVT) or metastasis portends a more ominous prognosis [10]. Dutta and colleagues from the Amrita Institute of Medical Sciences, Kochi, India, present their experience of SBRT with CyberKnife (CK) in 72 patients with $\mathrm{HCC}$ associated with PVT [11]. Etiology of HCC included alcohol, metabolic syndromes and viral hepatitis B and C. A third of patients achieved recanalization after SBRT with improved overall survival rates (actuarial overall survival in responders 14.4 months). The authors acknowledge the need for further data and suggest more studies including randomized trials comparing SBRT with sorafenib.

\section{Dental enamel defects and oral cavity manifestations in Asian patients with celiac disease}

Although celiac disease $(\mathrm{CeD})$ is mainly considered to affect the small intestine, extraintestinal and specifically oral manifestations are also recognized [12]. Data on dental and oral cavity manifestations of $\mathrm{CeD}$ are lacking. Ahmed and colleagues from the All India Institute of Medical Sciences, New Delhi, India, report the first Indian study of dental and oral manifestations in 118 patients (36 treatment naïve and 82 established on a gluten-free diet) with biopsy-proven $\mathrm{CeD}$ compared to 40 controls [13]. Significantly more patients with $\mathrm{CeD}(66.9 \%)$ had dental enamel defects (DED), recurrent aphthous ulcers (37.3\%) and xerostomia (68.6\%) compared to controls. The authors draw our attention to oral and dental manifestations of $\mathrm{CeD}$. Gastroenterologists should remember to assess the oral cavity (including teeth!) and indeed, dentists should also be aware of this finding as a potential sign of undetected $\mathrm{CeD}$.

\section{To assess the efficacy of safety measures adopted during endoscopy in the Corona Virus Disease-19 era for health care professionals-A single-centre tertiary care hospital experience}

Health care providers (HCPs) are at high risk for severe acute respiratory syndrome coronavirus-2 (SARS-COV- 
2) infection because of their contact with patients. HCPs working in the endoscopy units are at heightened risk from inhalation of airborne droplets [14]. Infection prevention and control have a dramatic effect in assuring the safety of both HCPs and patients, including (but not limited to) the availability and correct use of personal protective equipment (PPE), risk stratification of patients and interventions based on testing, separation and isolation of patients at high risk of Corona Virus Diease-19 (COVID-19). Ramchandani and colleagues from the Asian Institute of Gastroenterology, Hyderabad, India, report a retrospective experience of 3460 endoscopic procedures performed between 1 April and 31 July 2020 (34 on COVID-19 patients) and describe their detailed protocol for screening, risk stratification and safety measures for patients and HCPs [15]. Gastrointestinal bleeding (12/34) and biliary sepsis $(9 / 34)$ were the most common emergency/urgent indications. Symptomatic SARS-COV-2 infection was diagnosed in $6.5 \%(5 / 76) \mathrm{HCPs}$, all of whom recovered. Early implementation of protective mechanisms to prevent COVID-19 among HCPs on endoscopy units cannot be over-emphasized.

\section{Management of Helicobacter pylori infection in Indians: the Bhubaneswar Consensus Report of the Indian Society of Gastroenterology}

Helicobacter pylori remains a major source of gastroduodenal disease globally and particularly in India, where it is a major source of morbidity from peptic ulcer and gastric cancer [16]. The Indian Society of Gastroenterology publishes the 3rd consensus report on management of $H$. pylori in India in this issue of the Indian Journal of Gastroenterology [17]. This comprehensive document produced following a modified Delphi process provides consensus statements and a literature review of the epidemiology of $H$. pylori and associations, diagnosis, treatment and re-treatment after treatment failure, H. pylori and gastric cancer, prevention and public health concerns constituting essential reading for every practising gastroenterologist.

\section{Declarations}

Conflict of interest JKL declares no competing interests.

Disclaimer The author is solely responsible for the data and the contents of the paper. In no way, the Honorary Editor-in-Chief, Editorial Board Members, the Indian Society of Gastroenterology or the printer/ publishers are responsible for the results/findings and content of this article.

\section{References}

1. Burra P, Ferrarese A, Feltrin G. Quality of life and adherence in liver transplant recipients. Minerva Gastroenterol Dietol. 2018;64: 180-6.

2. Raju S, Mathew JS, Surendran S, Padma UD. Quality of life five years following liver transplantation. Indian J Gastroenterol. 2021;40. https://doi.org/10.1007/s12664-021-01180-w.

3. Nilsson E, Anderson H, Sargenti K, et al. Patients with liver cirrhosis show worse survival if decompensation occurs later during course of disease than at diagnosis. Scand J Gastroenterol. 2018;53:475-81.

4. Baiges A, Magaz M, Turon F, et al. Treatment of acute variceal bleeding in 2021-when to use transjugular intrahepatic portosystemic shunts? Clin Liver Dis. 2021;25:345-56.

5. Rajesh S, Philips CA, Betgeri SS, et al. Transjugular-intrahepaticportosystemic shunt placement at index portal hypertensive decompensation (anticipant-TIPS) in cirrhosis and the role of early intervention in variceal bleeding and ascites. Indian J Gastroenterol. 2021;40. https://doi.org/10.1007/s12664-021-01179-3.

6. Anand AC, Nandi B, Acharya SK, et al. INASL Task-Force on Acute Liver Failure. Indian National Association for the Study of the Liver Consensus Statement on Acute Liver Failure (Part 1): epidemiology, pathogenesis, presentation and prognosis. J Clin Exp Hepatol. 2020;10:339-76.

7. Govindarajan R, Ramamoorthy G, Shanmugam RM, et al. Rodenticide ingestion is an important cause of acute hepatotoxicity in Tamil Nadu, southern India. Indian J Gastroenterol. 2021;40. https://doi.org/10.1007/s12664-021-01178-4.

8. Targher G, Tilg H, Byrne CD. Non-alcoholic fatty liver disease: a multisystem disease requiring a multidisciplinary and holistic approach. Lancet Gastroenterol Hepatol. 2021;S2468-1253(21): $00020-0$.

9. Navarroza AMC, Wong SN. Comparison of clinical and metabolic profiles of lean versus non-lean nonalcoholic fatty liver disease. Indian J Gastroenterol. 2021;40. https://doi.org/10.1007/s12664021-01184-6.

10. Shampain KL, Hackett CE, Towfighi S, et al. SBRT for HCC: overview of technique and treatment response assessment. Abdom Radiol (NY). 2021. https://doi.org/10.1007/s00261-02103107-7.

11. Dutta D, Tatineni T, Gupte A, et al. Hepatocellular carcinoma patients with portal vein thrombosis treated with robotic radiosurgery: interim results of a prospective study. Indian J Gastroenterol. 2021;40. https://doi.org/10.1007/s12664-021-01172-w.

12. Al-Toma A, Volta U, Auricchio R, et al. European Society for the Study of Coeliac Disease (ESsCD) guideline for coeliac disease and other gluten-related disorders. United European Gastroenterol J. 2019;7:583-613.

13. Ahmed A, Singh A, Kajal S, et al. Dental enamel defects and oral cavity manifestations in Asian patients with celiac disease. Indian J Gastroenterol. 2021;40. https://doi.org/10.1007/s12664-02101175-7.

14. Ginès À, Fernández-Esparrach $\mathrm{G}$, Pellisé $\mathrm{M}$, et al. Critical importance of early introduction of prevention measures for SARS-CoV2 infection in endoscopy units. Gastrointest Endosc. 2020;92:9367.

15. Ramchandani M, Rughwani HR, Tevethia HV, et al. To assess the efficacy of safety measures adopted during endoscopy in the COVID-19 era for health care professionals - a single centre tertiary 
care hospital experience. Indian J Gastroenterol. 2021;40. https:// doi.org/10.1007/s12664-021-01146-y.

16. Proceedings of the 2nd National Workshop on Helicobacter pylori: management recommendations in India. Thrissur, Kerala, February 20, 1999. Indian J Gastroenterol. 2000;19 Suppl 1:1-40.

17. Singh SP, Ahuja V, Ghoshal UC, et al. Management of Helicobacter pylori infection in Indians: the Bhubaneswar
Consensus Report of the Indian Society of Gastroenterology. Indian J Gastroenterol. 2021;40. https://doi.org/10.1007/s12664021-01186-4.

Publisher's note Springer Nature remains neutral with regard to jurisdictional claims in published maps and institutional affiliations. 